\title{
Association of blood pressure with cognitive function at midlife: a Mendelian randomization study
}

\author{
Daokun Sun ${ }^{1}$, Emy A. Thomas ${ }^{1}$, Lenore J. Launer ${ }^{2}$, Stephen Sidney ${ }^{3}$, Kristine Yaffe ${ }^{4}$ and Myriam Fornage ${ }^{1,5^{*}}$ (D)
}

\begin{abstract}
Background: Whether high blood pressure has a causal effect on cognitive function as early as middle age is unclear. We investigated whether high blood pressure (BP) causally impairs cognitive function at midlife using Mendelian Randomization (MR).
\end{abstract}

Methods: We applied a two-sample MR approach to investigate the causal relationship between BP and midlife cognitive performance measured by the Digit Symbol Substitution Test (DSST), Rey Auditory Verbal Learning Test (RAVLT), and Stroop Interference test. We used a total of 109 genetic polymorphisms with established associations with BP as instrumental variables and estimated gene-cognitive function association in 1369 middle-aged adults (Mean age (SD): 50.8 (3.3), 54.0\% women) from the CARDIA study.

Results: A $10 \mathrm{mmHg}$ increment in genetically-predicted systolic, diastolic, or pulse pressure was associated with a 4.9 to 7.7 -point lower DSST score $(P=0.002$, SBP; $P=0.005$, DBP and $P=0.008$, PP), while a $10 \mathrm{mmHg}$ increment in genetically-predicted SBP was associated with a 0.7 point lower RAVLT and a 2.3 point higher Stroop $(P=0.046$ and 0.011 , respectively).

Conclusions: This MR analysis shows that high BP, especially SBP, is causally associated with poorer processing speed, verbal memory, and executive function during midlife. These findings emphasize the need for further investigation of the role and mechanisms of BP dysregulation on cognitive health in middle age and perhaps, more broadly, across the lifespan.

Keywords: Mendelian randomization, Blood pressure, Cognitive disorders, Risk factors, Dementia

\section{Background}

Hypertension is one of the long-established modifiable risk factors for age-related dementia [1]. Several studies have reported that high blood pressure (BP) developed by middle age is associated with cognitive decline $[2,3]$ and with global and domain-specific cognitive impairment

\footnotetext{
*Correspondence: myriam.fornage@uth.tmc.edu

${ }^{1}$ Brown Foundation Institute of Molecular Medicine, McGovern Medical School, The University of Texas Health Science Center at Houston, 1825 Pressler Street, Houston, TX 77030, USA

${ }^{5}$ Human Genetics Center, School of Public Health, The University of Texas Health Science Center at Houston, Houston, TX, USA

Full list of author information is available at the end of the article
}

in late life $[4,5]$. However, other studies have reported nonmonotonic or null relationships between high BP and latelife cognitive function [6,7]. The inconsistency of results may be due to differences in study populations, study design, and methodological aspects. Reverse causation may also play a role. Randomized clinical trials (RCTs) that examine the long-term effects of elevated blood pressure on cognitive ability would represent a gold standard but are costly and difficult to conduct since only randomization based on anti-hypertensive treatment rather than on BP level can be achieved. Early clinical trials designed to evaluate the cognitive benefits of antihypertensive treatment

(c) The Author(s). 2020 Open Access This article is licensed under a Creative Commons Attribution 4.0 International License, which permits use, sharing, adaptation, distribution and reproduction in any medium or format, as long as you give appropriate credit to the original author(s) and the source, provide a link to the Creative Commons licence, and indicate if changes were made. The images or other third party material in this article are included in the article's Creative Commons licence, unless indicated otherwise in a credit line to the material. If material is not included in the article's Creative Commons licence and your intended use is not permitted by statutory regulation or exceeds the permitted use, you will need to obtain permission directly from the copyright holder. To view a copy of this licence, visit http://creativecommons.org/licenses/by/4.0/ The Creative Commons Public Domain Dedication waiver (http://creativecommons.org/publicdomain/zero/1.0/) applies to the data made available in this article, unless otherwise stated in a credit line to the data. 
showed conflicting results, most of which were not in favor of beneficial effects [8]. The more recent SPRINT MIND trial reported that, in older adults at increased risk of cardiovascular disease, intensive systolic BP control reduced the risk of mild cognitive impairment (MCI) as well as the combined occurrence of MCI or dementia [9]. Whether the possible benefits of BP control in older adults extend earlier in life is unknown.

We employed a two-sample MR approach to expand existing knowledge about the effect of high BP burden on cognitive health during middle age [10], a pivotal period in the life course when cognitive function begins to decline among healthy adults [11].

\section{Methods}

\section{Study population}

Participants were from the Coronary Artery Risk Development in Young Adults (CARDIA) study, a prospective multi-center cohort study investigating the natural history and etiology of cardiovascular disease in 2637 AfricanAmericans and 2478 European-Americans aged 18-30 years at the time of initial examination in 1985-1986 [12]. Participants were recruited from the community in Birmingham, AL; from selected census tracts in Chicago, IL and Minneapolis, MN; and from the Kaiser-Permanente health plan membership in Oakland, CA. The sample was approximately balanced with respect to race, age, sex, and education groups. Participants have completed nine sequential examinations every 2 to 5 years for 30 years. For this study, we used 1369 subjects of European ancestry who had genome-wide genotype data and phenotypic data for three cognitive tests at Year 25 examination.

\section{Cognitive assessment}

Three standardized tests in CARDIA were administered at Year 25 examination (2010-2011) to assess major aspects of cognitive function by trained and certified technicians following an established protocol as described elsewhere [13] and in the supplemental materials (Additional File 1). Briefly, the battery of tests included the Rey Auditory Verbal Learning Test (RAVLT) (score range: 0-15), a measure of verbal learning and memory [14]; the Digit Symbol Substitution Test (DSST) (Part D) (score range: 0133), a measure primarily of psychomotor speed; and the Stroop Interference Test (score range: 1-160), a measure of executive function [15]. A higher score in DSST and RAVL $\mathrm{T}$ indicates better cognition in the corresponding domain whereas that in the Stroop Interference test reflects poor cognitive performance.

\section{Instrumental variables and association analysis}

A total of 152 single nucleotide polymorphisms (SNPs) previously identified in one of the largest GWAS of BP in individuals of European descent [16] were selected for analysis. These SNPS were selected based on their genome-wide significant association with blood pressure traits $\left(P<5 \times 10^{-8}\right)$ and evidence of independent replication in multiple studies. From the summary statistics, we obtained the association estimate of each SNP with systolic blood pressure (SBP), diastolic blood pressure (DBP), and pulse pressure (PP) respectively, which had been replicated among 140,886 European participants. Of these, 145 were available in CARDIA. To minimize pleiotropic effects [17], we removed 8 SNPs which overlapped or were in high linkage disequilibrium (LD) $\left(\mathrm{r}^{2}>\right.$ $0.8)$ with SNPs influencing either known BP risk factors (body mass index (BMI), smoking, and type 2 diabetes), which can serve as confounders; or education, which may bias the gene-cognition association [18]. We also pruned SNPs that were in LD with one another at $r^{2}$ greater than 0.001 within a $10 \mathrm{~kb}$ distance. Thus, we used 109 SNPs independently and exclusively associated with BP at genome-wide significance level as instrumental variables (IVs) (99 for SBP, 98 for DBP, and 98 for PP, Table S1). We estimated the allelic effect of each SNP on measures of cognitive performance in 1369 CARDIA participants adjusting for age, sex, and study site. We aligned the direction of SNP-cognitive function association with that in the BP GWAS.

\section{Statistical analysis}

We implemented a multivariable two-sample Mendelian Randomization (MR) approach using the TwoSampleMR $\mathrm{R}$ package [19] to explore the causal association of genetically-predicted BPs with DSST, RAVLT, and Stroop test's performance. A summary of our analytical approach is shown in Figure S1 (Additional file 1). As primary analysis, we employed a standard inverse-variance weighted (IVW) method to derive the causal estimates for each variant, which were combined by random-effects metaanalysis. We carried out detection of potentially invalid or influential instruments using radial plots in RadialMR [20]. We then repeated IVW MR analyses after removing the outliers. We applied additional MR methods including the weighted median method [21], MR-Egger regression [22], and the contamination mixture MR [23] as sensitivity analyses for robust inference.

To examine the effect of potential unbalanced pleiotropy on causal estimates, we employed the MR-Egger intercept test [24], the Mendelian Randomization Pleiotropy RESidual Sum and Outlier (MRPRESSO) test [25] and constructed funnel plots [26]. We evaluated the assumption of "NO Measurement Error" (NOME) by Bowden $I^{2}$ statistics. We further estimated the heterogeneity of selected IVs by Cochran's Q test [27]. To preclude the possibility that the causal inference was driven by any individual variant, we performed a leave-one-out analysis for the IVW and MR-Egger methods. 
Because of the overlap in genetic instruments for the 3 BP measures (Table S1, Additional file 1) and correlations among cognitive tests, we did not adjust for multiple testing. A $P$-value of association less than 0.05 is considered significant.

\section{Results}

The CARDIA participants were in their fifties (Mean [range] age: 51 [42-57] years) at Year 25. They were well educated but were slightly overweight. One-quarter of them were hypertensive, but less than one fifth were taking anti-hypertension medication. The prevalence of type 2 diabetes and current smoking were low (Table 1).

The most notable association by the standard IVW method was found between midlife genetically-predicted BP level and DSST score. For each unit ( $\mathrm{mm} \mathrm{Hg}$ ) increment in genetically-predicted BP level, there was a 0.49 to 0.67-point lower DSST score. There was no significant association between genetically-predicted BPs and performance on the RAVLT or Stroop Interference test. The direction of association was however consistent among the 3 cognitive tests (Table 2).

Table 1 Characteristics of 1369 CARDIA participants with cognitive function at Year 25

\begin{tabular}{|c|c|}
\hline Variable & Value \\
\hline Sample size, N & 1369 \\
\hline Age (SD) (year) & $50.8(3.3)$ \\
\hline Female (\%) & $739(54.0)$ \\
\hline \multicolumn{2}{|l|}{ Center (\%) } \\
\hline Birmingham, AL & $273(19.9)$ \\
\hline Chicago, IL & $320(23.4)$ \\
\hline Minneapolis, MN & $443(32.4)$ \\
\hline Oakland, CA & $333(24.3)$ \\
\hline At least college education (\%) & $917(67.0)$ \\
\hline BMI (SD) $\left(\mathrm{kg} / \mathrm{m}^{2}\right)$ & $28.3(6.1)$ \\
\hline Type2 Diabetes (\%) & $124(9.1)$ \\
\hline Current Smoking (\%) & $165(12.1)$ \\
\hline Systolic Blood Pressure (SD) (mmHg) & $114.4(13.7)$ \\
\hline Diastolic Blood Pressure (SD) (mmHg) & $70.8(10.1)$ \\
\hline Pulse Pressure (SD) (mmHg) & $43.6(7.6)$ \\
\hline Hypertension (\%) & $341(24.9)$ \\
\hline HBP Rx use (\%) & $228(16.7)$ \\
\hline DSST (Mean (SD): Min-Max) & 75.0 (14.9): $21-125$ \\
\hline RAVLT (Mean (SD): Min-Max) & $9.4(3.1): 0-15$ \\
\hline Stroop (Mean (SD): Min-Max) & 19.3 (8.0): 4-71 \\
\hline
\end{tabular}

Abbreviations: CARDIA, the Coronary Artery Risk Development in Young Adults Study; SD Standard deviation, $B M I$ Body mass index (calculated as weight in kilograms divided by height in meters squared), $H B P R x$ Anti-hypertensive medication, DSST Digit Symbol Substitution Test, RAVLT Rey Auditory Verbal Learning Test, Stroop The Stroop Interference test
We conducted analyses to detect potential invalid and/ or influential instruments. Radial plots indicated the presence of outlier instruments (Figure S2 in Additional file 1). After removing these outliers, the causal associations between genetically-predicted BPs and midlife cognitive function were generally strengthened (Table 2). Notably, genetically-predicted SBP was now also associated with RAVLT and Stroop performance. Each unit $(\mathrm{mm} \mathrm{Hg})$ increment in genetically-predicted SBP level was associated with a 0.07-point lower RAVLT score and a 0.23 -point higher Stroop Interference test score (Table 2).

The IVW method is optimally efficient when all genetic variants are valid IVs but is biased when one or more genetic variants are invalid IVs. Therefore, we performed sensitivity analyses using additional MR methods that vary in their robustness to the presence of invalid IVs (Tables 3 and 4). The weighted median method indicated a similar or stronger magnitude of causal associations, although with larger confidence intervals, while the MR-Egger method was insufficiently powered. Estimates from the contamination mixture MR method were similar to those of the IVW (Tables 3 and 4). The Bowden $I^{2}$ statistics across the three BP exposures were all high, around 0.96, indicating a high strength of IVs and a less than moderate degree of NOME assumption violation (Tables 3 and 4). The mean F-statistics, another measure of IV strength, were 29.4 for SBP, 32.8 for DBP, and 26.0 for PP, indicating that our causal estimates are not likely to be biased by weak IVs.

We also conducted sensitivity analyses to investigate potential pleiotropy, which could invalidate MR assumptions and bias causal estimates. Indeed, a key assumption of $M R$ is that the IVs have an effect on the outcome (cognition) only via the exposure (BP). Using the MREgger intercept test, we did not detect evidence of significant directional pleiotropy in any of the analyses performed. Consistently, the MR-PRESSO global test also did not indicate any evidence of directional pleiotropy (Tables 3 and 4). We also plotted IV strength against IV estimates in funnel plots (Figure S3) and confirmed the absence of pleiotropy. All of these plots showed a symmetrical distribution indicating that pleiotropic effects were well balanced across all the genetic variants. Cochran $Q$ statistics also confirmed that there was no evidence of heterogeneity in causal estimates (Tables 3 and 4).

To further preclude the possibility that the causal inference was driven by any individual variant, we performed additional sensitivity analyses. Leave-one-out results confirmed that the causal estimates were not driven by the influence of any individual SNP (Figures S4 and S5). Indeed, while omitting some variants placed heavier burden on causal estimates than others, these 


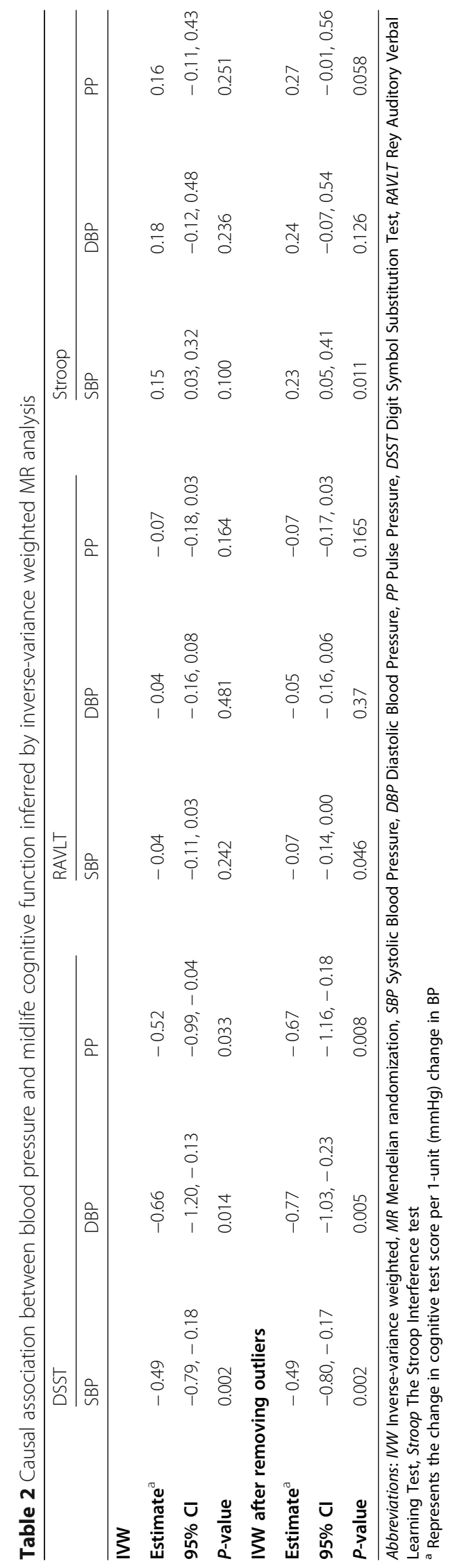




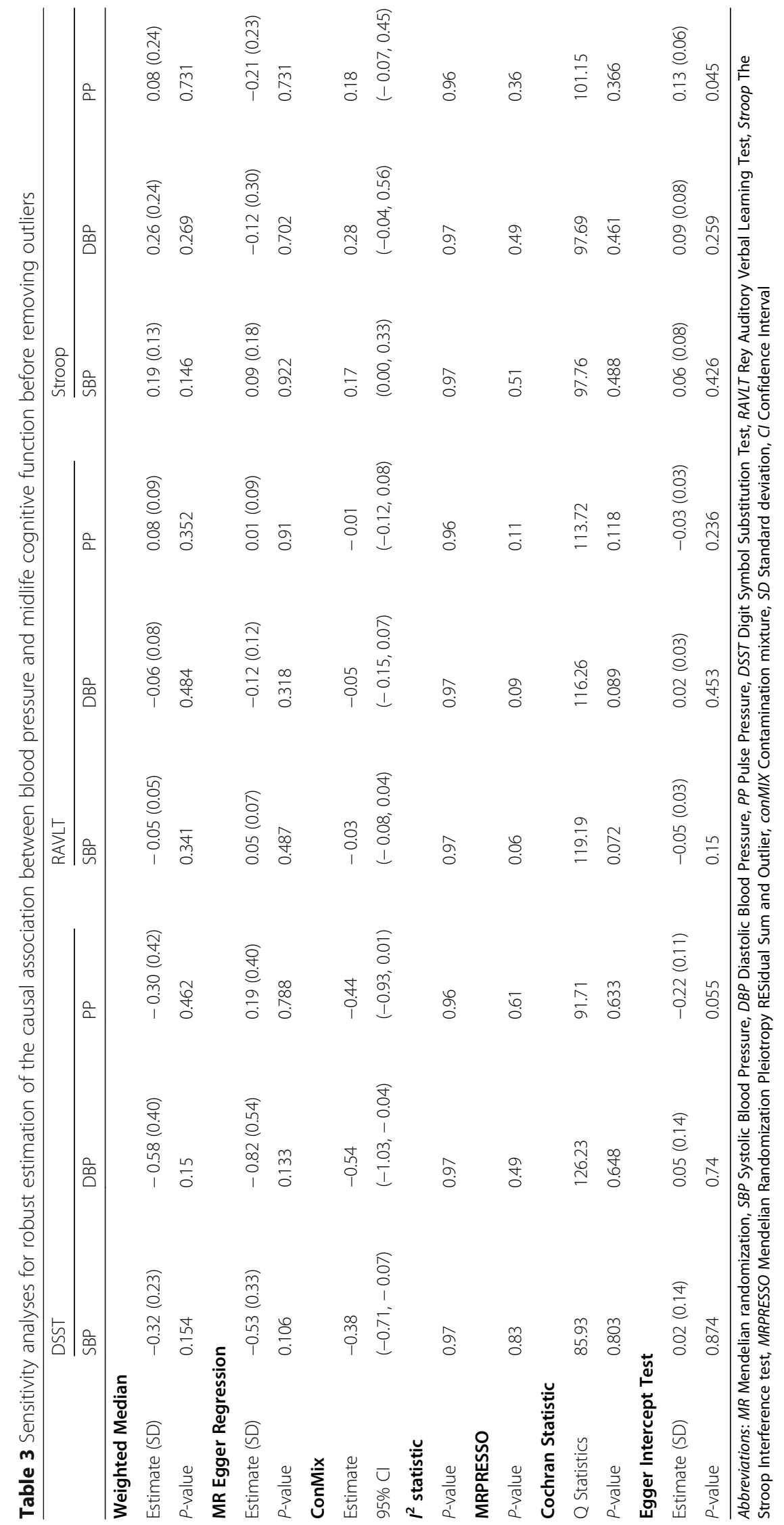




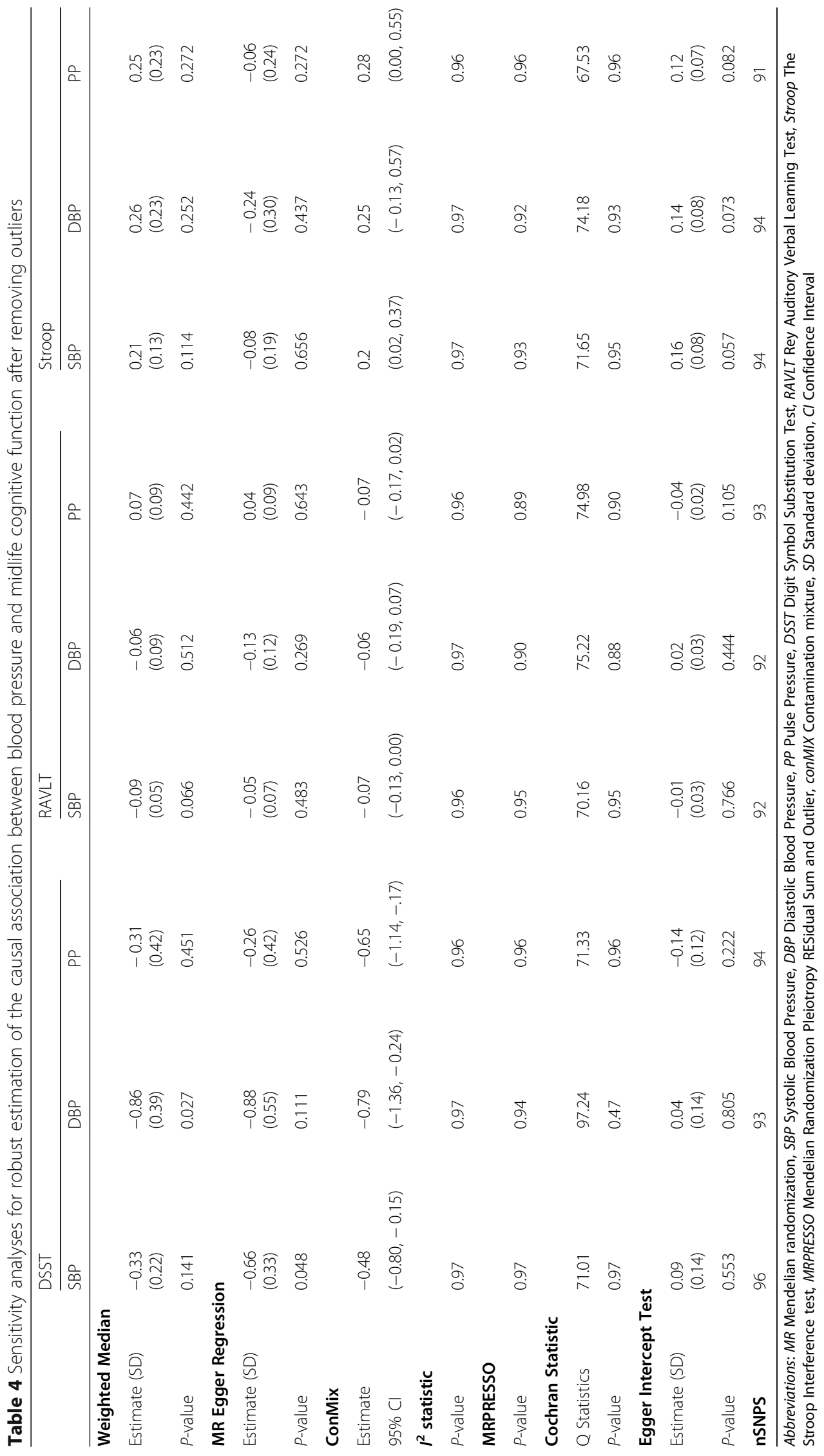


estimates were, both, stable and consistent in direction (did not cross the 0 line).

\section{Discussion}

Mendelian randomization holds substantial promise for causal inference from observational data. This approach mimics the random allocation in RCTs by using risk factor-associated genetic variants as IVs. It is based on the principle that the distribution of those genetic variants in the population is randomly assigned at meiosis, independent of potential confounders and not susceptible to reverse causation or other biases [17]. In this study, we inferred the causal effect of high BP on midlife cognitive function through a two-sample MR method and further sensitivity analyses. We report that higher genetically-predicted BP, especially SBP, is causally associated with lower midlife processing speed and lower executive function and verbal memory but the estimated effects of genetically-predicted BP on midlife cognitive function is small.

A recent MR study from the UK Biobank and including participants aged 40 to 70 years observed no detrimental effect of genetically-predicted SBP on cognitive ability measured by a verbal-numerical reasoning test [28]. Given the differences in study design, including cognitive assessment and participant characteristics, it is possible that one does not contradict the other. Indeed, compared to our study, the UK Biobank study included a large range in population age. Including older individuals into the analysis has the potential to introduce biases owing to the effect of high blood pressure on mortality [29, 30]. Moreover, the relationship between BP and cognitive function may vary across the lifespan. For example, a MR study recently reported that higher genetically-predicted SBP significantly lowered the risk of Alzheimer's disease [31].

The specific mechanisms that underlie the causal association between high BP and poor processing speed are not known. One possible mechanism may involve the known pathologies of hypertension-induced brain vascular injury, most notably to the cerebral small vessels. Indeed, long-term hypertension is known to cause vascular hypertrophy and microvascular remodeling, which result in regional cerebral blood flow dysfunction and lead to white matter disease and neuronal loss [32]. Indeed, previous studies have suggested that elevated BP is a major risk factor for several magnetic resonance imaging (MRI) markers of cerebral small vessel disease (SVDs) [33]. For example, elevated SBP is associated with brain atrophy, reduced gray matter volume and white matter hyperintensities (WMH) [34], while DBP is associated with brain atrophy and WMH [35]. It has been shown that the presence of SVD appears to align with a compromised cognitive profile of early-impaired processing speed [36, 37]. Elevated BP may also affect brain anatomical connectivity. Indeed, regional white matter integrity is lower among individuals with higher $\mathrm{BP}$, regardless of hypertension status [38]. In a recent study of cognitively healthy older adults, the relationship between SBP and poorer processing speed appeared to be mediated by functional connectivity of the right superior temporal gyrus within the ventral attention network (VAN) [39]. This is consistent with a previous report showing that variation in structural organization within the frontoparietal system, which comprises the VAN, is associated with differences in attentional functions, including visual short-term memory capacity, processing speed, and spatial bias [40].

Whether antihypertensive treatment can reverse the pathological process of vascular damage, restore cerebral function, and improve cognitive ability remains inconclusive. On one hand, it appears that lowering BP is helpful to rehabilitate some of the vascular functions impaired by BP elevation. For instance, a previous clinical trial among subjects older than 70 suggested that right-shifted cerebral autoregulation curve induced by hypertension can be back-shifted towards normal through intensive BP lowering [41]. On the other hand, however, most clinical trials have failed to identify significant benefits of antihypertensive therapy in improving cognitive fitness [8]. The majority of these studies were carried out over a short period of time and limited to aged populations on average between 62 to 83 years of age. Elderly individuals may be exposed to the negative effects of hypertension for a longer time and may have more severe vascular injury due to hypertension than middle-aged adults, and, thus, may be more resistant to the benefits of $\mathrm{BP}$ lowering. Moreover, the effects of antihypertensive treatment on cognitive function or risk of dementia have been shown to differ across therapeutic classes [42], suggesting that the neuroprotective effects of antihypertensive therapy may extend beyond BP lowering.

Strengths of our study include the use of a multi-SNP genetic instrument with well-established associations with BP. The largest BP GWAS at the time of this analysis reported genetic association for 267 SNPs in total, 115 of which were newly validated [16]. We opted for the smaller set of SNPs, which were previously identified with stronger evidence of validity, although fewer IVs theoretically reduce statistical power [43]. The use of summary data for gene-BP association from a large GWAS analysis also improved power and precision of our study. However, several limitations are acknowledged. First, the genetic variants for the various BP measures almost fully overlapped, which makes it difficult to discriminate possible unique causal relationships between the specific components of BP and cognitive function. Excluding genetic variants that overlap among BP traits would likely result in a loss of strength of 
our MR analyses. Second, possible effects of unmeasured confounders are a recognized limitation of the MR approach. In particular, the possible confounding effects of antihypertensive medication use could not be accounted for in our analyses because the estimates of allelic effects on BP were obtained from a GWAS that corrected for medication use by adding 15 and $10 \mathrm{mmHg}$ to the SBP and DBP values, respectively. If genetically predicted higher $\mathrm{BP}$ is also associated with a higher probability of being on antihypertensive medication as previously observed [31], the true causal association between $\mathrm{BP}$ and cognitive function is likely underestimated. Moreover, the GWAS summary statistics used in this analysis adjusted for BMI and, thus, can potentially yield biased estimates. However, a recent study using both simulations and empirical data from the UK Biobank showed that using adjusted and corrected GWAS in MR analysis is unlikely to make a large difference to causal estimates [44]. Third, these analyses were conducted in persons of European ancestry. Whether the results are generalizable to diverse populations remains to be examined. Expanding efforts for genetic discoveries of BP in African-Americans and Hispanics/Latinos is required to meet this need. Finally, although this study provided evidence for a causal relationship between SBP and processing speed, it does not shed light on the possible biological mechanisms involved.

\section{Conclusion}

By providing support for a causal relationship between $\mathrm{BP}$ and cognitive health in middle age, our MR study underscores the need for further investigations of the role and mechanisms of $\mathrm{BP}$ dysfunction on cognitive health across the lifespan, which may inform on early intervention and timely treatment of hypertension to maintain brain health.

\section{Supplementary information}

Supplementary information accompanies this paper at https://doi.org/10. 1186/s12920-020-00769-y.

\section{Additional file 1: Cognitive assessment in CARDIA at Year 25}

examination (2010-2011); Table S1. SNPs and SNP-exposure effects used in the MR analyses.; Figure S1. Summary of the MR approach used in the study. Figure S2. Radial plot for detection of outliers. Figure S3. Funnel plot of blood pressure effect on cognitive function. Figure S4. Leave-one-out result of IWW estimates. Figure S5. Leave-one-out result of MR-Egger estimates.

\section{Abbreviations}

BP: Blood Pressure; BMI: Body Mass Index; CARDIA: Coronary Artery Risk Development in Young Adults; DBP: Diastolic Blood Pressure; DSST: Digit Symbol Substitution Test; GWAS: Genome-wide Association Study; IV: Instrumental Variable; IMW: Inverse-Variance Weighted; LD: Linkage Disequilibrium; MCI: Mild Cognitive Impairment; MR: Mendelian Randomization; NOME: NO Measurement Error; PP: Pulse Pressure; RAVLT: Rey Auditory Verbal Learning Test; RCT: Randomized Controlled Trial; SBP: Systolic Blood Pressure; SD: Standard Deviation; SNP: Single Nucleotide
Polymorphism; SVD: Cerebral Small Vessel Disease; VAN: Ventral Attention Network; WMH: White Matter Hyperintensities

\section{Acknowledgments}

The Coronary Artery Risk Development in Young Adults Study (CARDIA) is conducted and supported by the National Heart, Lung, and Blood Institute (NHLBI) in collaboration with the University of Alabama at Birmingham (HHSN268201300025I \& HHSN268201300026I), Northwestern University (HHSN268201300027I), University of Minnesota (HHSN268201300028I), Kaiser Foundation Research Institute (HHSN268201300029l), and Johns Hopkins University School of Medicine (HHSN268200900041I). CARDIA is also partially supported by the Intramural Research Program of the National Institute on Aging (NIA) and an intra-agency agreement between NIA and NHLBI (AG0005). Genotype data were obtained as part of the Gene Environment Association Studies (GENEVA) through grants U01-HG004729, U01-HG04424, and U01-HG004446 from the National Human Genome Research Institute. This manuscript has been reviewed by CARDIA for scientific content.

\section{Authors' contributions}

DS performed the statistical analyses, interpreted the data, and was a major contributor in writing the manuscript; EAT performed the statistical analyses; $L J$, SS, and KY supported the collection of the phenotypic data; MF conceived and designed the study, supported the collection of the genetic data, and drafted the manuscript. All authors read and approved the manuscript.

\section{Funding}

This work was supported in part by grants from the National Institutes of Health R01 HL122658 and NS087541.

\section{Availability of data and materials}

CARDIA data are available through the National Center for Biotechnology Information database of Genotypes and Phenotypes (dbGaP). More information is provided at https://www.cardia.dopm.uab.edu/studyinformation/genetic-data/cardia-genetic-data-in-dbgap-ncbi.

\section{Ethics approval and consent to participate}

Written informed consent was obtained from participant at each examination and all study protocols were approved by the institutional review boards of the University of Texas Health Science Center at Houston, the University of Alabama at Birmingham, the University of Minnesota, Northwestern University, and the Kaiser Permanente Division of Research.

\section{Consent for publication}

Not applicable.

\section{Competing interests}

The authors declare that they have no competing interests.

\section{Author details}

${ }^{1}$ Brown Foundation Institute of Molecular Medicine, McGovern Medical School, The University of Texas Health Science Center at Houston, 1825 Pressler Street, Houston, TX 77030, USA. ' Laboratory of Epidemiology and Population Science, National Institute on Aging, National Institutes of Health, Bethesda, MD, USA. ${ }^{3}$ Kaiser Permanente Northern California Division of Research, Oakland, CA, USA. ${ }^{4}$ Departments of Psychiatry and Neurology, University of California San Francisco School of Medicine, San Francisco, CA, USA. ${ }^{5}$ Human Genetics Center, School of Public Health, The University of Texas Health Science Center at Houston, Houston, TX, USA

Received: 12 May 2020 Accepted: 17 August 2020

Published online: 26 August 2020

\section{References}

1. Livingston G, Sommerlad A, Orgeta V, Costafreda SG, Huntley J, Ames D, Ballard C, Banerjee S, Burns A, Cohen-Mansfield J, et al. Dementia prevention, intervention, and care. Lancet. 2017;390(10113):2673-734.

2. Gottesman RF, Schneider AL, Albert M, Alonso A, Bandeen-Roche K, Coker L, Coresh J, Knopman D, Power MC, Rawlings A, et al. Midlife hypertension and 20-year cognitive change: the atherosclerosis risk in communities neurocognitive study. JAMA Neurol. 2014;71(10):1218-27. 
3. Debette S, Seshadri S, Beiser A, Au R, Himali JJ, Palumbo C, Wolf PA, DeCarli C. Midlife vascular risk factor exposure accelerates structural brain aging and cognitive decline. Neurology. 2011;77(5):461-8.

4. Elias MF, Wolf PA, D'Agostino RB, Cobb J, White LR. Untreated blood pressure level is inversely related to cognitive functioning: the Framingham study. Am J Epidemiol. 1993;138(6):353-64.

5. Launer LJ, Masaki K, Petrovitch H, Foley D, Havlik RJ. The association between midlife blood pressure levels and late-life cognitive function. The Honolulu-Asia Aging Study. Jama. 1995;274(23):1846-51.

6. Hebert LE, Scherr PA, Bennett DA, Bienias JL, Wilson RS, Morris MC, Evans DA. Blood pressure and late-life cognitive function change: a biracial longitudinal population study. Neurology. 2004;62(11):2021-4.

7. Taylor C, Tillin T, Chaturvedi N, Dewey M, Ferri CP, Hughes A, Prince M, Richards M, Shah A, Stewart R. Midlife hypertensive status and cognitive function 20 years later: the Southall and Brent revisited study. J Am Geriatr Soc. 2013;61(9):1489-98.

8. Iadecola C, Yaffe K, Biller J, Bratzke LC, Faraci FM, Gorelick PB, Gulati M Kamel H, Knopman DS, Launer LJ, et al. Impact of hypertension on cognitive function: a scientific statement from the American Heart Association. Hypertension. 2016;68(6):e67-94.

9. Sprint Mind Investigators for the SPRINT Research Group, Williamson JD, Pajewski NM, Auchus AP, Bryan RN, Chelune G, Cheung AK, Cleveland ML, Coker LH, Crowe MG, et al. Effect of intensive vs standard blood pressure control on probable dementia: a randomized clinical trial. Jama. 2019;321(6): 553-61.

10. Yaffe K, Vittinghoff E, Pletcher MJ, Hoang TD, Launer L, Whitmer R, Coker LH, Sidney S. Early adult to midlife cardiovascular risk factors and cognitive function. Circulation. 2014;129(15):1560-7.

11. Singh-Manoux A, Kivimaki M, Glymour MM, Elbaz A, Berr C, Ebmeier KP, Ferrie JE, Dugravot A. Timing of onset of cognitive decline: results from Whitehall II prospective cohort study. Bmj. 2012;344:d7622.

12. Friedman GD, Cutter GR, Donahue RP, Hughes GH, Hulley SB, Jacobs DR Jr, Liu K, Savage PJ. CARDIA: study design, recruitment, and some characteristics of the examined subjects. J Clin Epidemiol. 1988;41(11): 1105-16.

13. Launer LJ, Miller ME, Williamson JD, Lazar RM, Gerstein HC, Murray AM, Sullivan M, Horowitz KR, Ding J, Marcovina S, et al. Effects of intensive glucose lowering on brain structure and function in people with type 2 diabetes (ACCORD MIND): a randomised open-label substudy. Lancet Neurol. 2011;10(11):969-77.

14. Rosenberg SJ, Ryan JJ, Prifitera A. Rey auditory-verbal learning test performance of patients with and without memory impairment. J Clin Psychol. 1984;40(3):785-7.

15. Stroop JR. Studies of interference in serial verbal reactions. J Exp Psychol. 1935;18(6):643.

16. Warren HR, Evangelou E, Cabrera CP, Gao H, Ren M, Mifsud B, Ntalla I, Surendran P, Liu C, Cook JP, et al. Genome-wide association analysis identifies novel blood pressure loci and offers biological insights into cardiovascular risk. Nat Genet. 2017:49(3):403-15.

17. Davey Smith G, Hemani G. Mendelian randomization: genetic anchors for causal inference in epidemiological studies. Hum Mol Genet. 2014;23(R1):R89-98.

18. Buniello A, MacArthur JAL, Cerezo M, Harris LW, Hayhurst J, Malangone C, McMahon A, Morales J, Mountjoy E, Sollis E, et al. The NHGRI-EBI GWAS catalog of published genome-wide association studies, targeted arrays and summary statistics 2019. Nucleic Acids Res. 2019;47(D1):D1005-12.

19. Hemani G, Zheng J, Elsworth B, Wade KH, Haberland V, Baird D, Laurin C, Burgess S, Bowden J, Langdon R, et al. The MR-base platform supports systematic causal inference across the human phenome. Elife. 2018;7:e34408.

20. Bowden J, Spiller W, Del Greco MF, Sheehan N, Thompson J, Minelli C, Davey Smith G. Improving the visualization, interpretation and analysis of two-sample summary data Mendelian randomization via the radial plot and radial regression. Int J Epidemiol. 2018;47(6):2100.

21. Bowden J, Davey Smith G, Haycock PC, Burgess S. Consistent estimation in Mendelian randomization with some invalid instruments using a weighted median estimator. Genet Epidemiol. 2016;40(4):304-14.

22. Bowden J, Davey Smith G, Burgess S. Mendelian randomization with invalid instruments: effect estimation and bias detection through egger regression. Int J Epidemiol. 2015;44(2):512-25.

23. Burgess S, Foley CN, Allara E, Staley JR, Howson JMM. A robust and efficient method for Mendelian randomization with hundreds of genetic variants. Nat Commun. 2020;11(1):376.
24. Burgess S, Thompson SG. Interpreting findings from Mendelian randomization using the MR-egger method. Eur J Epidemiol. 2017;32(5): 377-89.

25. Verbanck $M$, Chen $C Y$, Neale B, Do R. Detection of widespread horizontal pleiotropy in causal relationships inferred from Mendelian randomization between complex traits and diseases. Nat Genet. 2018;50(5):693-8.

26. Burgess S, Bowden J, Dudbridge F, Thompson SG. Robust instrumental variable methods using multiple candidate instruments with application to Mendelian randomization. In: ARXIV. eprint arXiv:1606.03729; 2016.

27. Burgess S, Bowden J, Fall T, Ingelsson E, Thompson SG. Sensitivity analyses for robust causal inference from Mendelian randomization analyses with multiple genetic variants. Epidemiology. 2017;28(1):30-42.

28. Hagenaars SP, Gale CR, Deary IJ, Harris SE. Cognitive ability and physical health: a Mendelian randomization study. Sci Rep. 2017;7(1):2651.

29. Boef AG, le Cessie S, Dekkers OM. Mendelian randomization studies in the elderly. Epidemiology. 2015;26(2):e15-6.

30. Vansteelandt S, Dukes O, Martinussen T. Survivor bias in Mendelian randomization analysis. Biostatistics. 2018;19(4):426-43.

31. Ostergaard SD, Mukherjee S, Sharp SJ, Proitsi P, Lotta LA, Day F, Perry JR, Boehme KL, Walter S, Kauwe JS, et al. Associations between Potentially Modifiable Risk Factors and Alzheimer Disease: A Mendelian Randomization Study. PLoS Med. 2015;12(6):e1001841 discussion e1001841.

32. Pantoni $L$, Simoni M. Pathophysiology of cerebral small vessels in vascular cognitive impairment. Int Psychogeriatr. 2003;15(Suppl 1):59-65.

33. Smith EE, Beaudin AE. New insights into cerebral small vessel disease and vascular cognitive impairment from MRI. Curr Opin Neurol. 2018;31(1):36-43.

34. Maillard P, Seshadri S, Beiser A, Himali JJ, Au R, Fletcher E, Carmichael O, Wolf PA, DeCarli C. Effects of systolic blood pressure on white-matter integrity in young adults in the Framingham heart study: a cross-sectional study. Lancet Neurol. 2012;11(12):1039-47.

35. McNeil CJ, Myint PK, Sandu AL, Potter JF, Staff R, Whalley $\sqcup$, Murray AD. Increased diastolic blood pressure is associated with MRI biomarkers of dementia-related brain pathology in normative ageing. Age Ageing. 2018;47(1):95-100.

36. Prins ND, van Dijk EJ, den Heijer T, Vermeer SE, Jolles J, Koudstaal PJ, Hofman A, Breteler MM. Cerebral small-vessel disease and decline in information processing speed, executive function and memory. Brain. 2005;128(Pt 9):2034-41.

37. Lawrence AJ, Patel B, Morris RG, MacKinnon AD, Rich PM, Barrick TR, Markus HS. Mechanisms of cognitive impairment in cerebral small vessel disease: multimodal MRI results from the St George's cognition and neuroimaging in stroke (SCANS) study. PLoS One. 2013;8(4):e61014.

38. Salat DH, Williams VJ, Leritz EC, Schnyer DM, Rudolph JL, Lipsitz LA, McGlinchey RE, Milberg WP. Inter-individual variation in blood pressure is associated with regional white matter integrity in generally healthy older adults. Neurolmage. 2012;59(1):181-92.

39. Wong NML, Ma EP, Lee TMC. The integrity of the Corpus callosum mitigates the impact of blood pressure on the ventral attention network and information processing speed in healthy adults. Front Aging Neurosci. 2017;9:108.

40. Chechlacz M, Gillebert CR, Vangkilde SA, Petersen A, Humphreys GW. Structural variability within Frontoparietal networks and individual differences in Attentional functions: an approach using the theory of visual attention. J Neurosci. 2015;35(30):10647-58.

41. Tryambake D, He J, Firbank MJ, O'Brien JT, Blamire AM, Ford GA. Intensive blood pressure lowering increases cerebral blood flow in older subjects with hypertension. Hypertension. 2013;61(6):1309-15.

42. Levi Marpillat N, Macquin-Mavier I, Tropeano Al, Bachoud-Levi AC, Maison P. Antihypertensive classes, cognitive decline and incidence of dementia: a network meta-analysis. J Hypertens. 2013;31(6):1073-82.

43. Pierce BL, Ahsan H, Vanderweele TJ. Power and instrument strength requirements for Mendelian randomization studies using multiple genetic variants. Int J Epidemiol. 2011;40(3):740-52.

44. Walker VM, Harrison S, Carter AR, Gill D, Tzoulaki I, Davies NM. The consequences of adjustment, correction and selection in genome-wide association studies used for two-sample Mendelian randomization. medRxiv. 2020; 2020.2007.2013.20152413. https://doi.org/10.1101/2020.07.13.20152413.

\section{Publisher's Note}

Springer Nature remains neutral with regard to jurisdictional claims in published maps and institutional affiliations. 